On autocar headlights

This content has been downloaded from IOPscience. Please scroll down to see the full text. 1922 Trans. Opt. Soc. 23270

(http://iopscience.iop.org/1475-4878/23/4/307)

View the table of contents for this issue, or go to the journal homepage for more

Download details:

IP Address: 128.192.114.19

This content was downloaded on 10/09/2015 at 18:28

Please note that terms and conditions apply. 


\title{
ON AUTOCAR HEADLIGHTS
}

\author{
BY FR. MEYER. \\ (Messrs Carl Zeiss, Jena.)
}

THE question as to whether a motorist should be at liberty to use headlights liable to dazzle pedestrians or the occupants of vehicles travelling in the opposite direction has been discussed so often that it would almost seem superfluous to say any more about it. Yet, the views of the two parties concerned-the motorist and the public -are as divergent as those of landlords and tenants under the blessings of an act which under one form of illumination presents itself as rent restriction, under another as tenancy extension. The fact is, the motorist wants a light which will reach as far ahead as possible, so as to enable him to anticipate any obstacle in his way and to stop his high-speed car in good time, if necessary. Obviously, facilities for driving a car at night at speeds which are attainable in day time add very materially to its value. Many business men prefer the late hours of the evening for travelling, so as to keep the entire day free for business; and, naturally, an hour saved by fast travelling and secured for rest is not to be despised.

This, however, can only be achieved with bright and therefore somewhat dazzling headlights of the searchlight type. The public, on the other hand, looks upon these powerful flaring lights as a threat to its safety, and in a sense it is justified in so doing. It would be a monstrous affront to the chivalry of the road if in much frequented places every car were to light up its track with glaring headlights, the more so as very deplorable accidents have occurred from this very cause. In the majority of cases, however, the blame for the accident lies with the public. If, instead of turning their backs to the approaching car and stepping out of the beam of light in the brightly illuminated street or road, a pedestrian faces the car and its dazzling lights and then frantically leaps from one side to the other, he places the driver of the car in an almost hopeless position. Many accidents could have been avoided if pedestrians would remember that a motorist does not deliberately run over anyone who is steadily walking along the road and whom he can see. I do not suggest, of course, that the indiscriminate use of headlights is to be advocated, or that it is justifiable in inhabited localities. On the other hand it does no good to forbid altogether the use of bright headlights, even though they dazzle, as has been done in some countries. In some cases no headlights are permissible which throw a beam above a certain height, say 39 to 48 inches above the ground. This is a regulation which seriously cripples a projector as a means of lighting up a long way ahead. For, in every projector, no matter how well designed, the comparatively short focal length of its optical component inevitably gives rise to dispersion of the beam through several degrees in upward and downward directions. Dipping the headlight sufficiently to prevent the bright beam from rising more than 39 to 48 inches above the ground will cause its central shaft to meet the ground only a few yards in front of the car, where it lights up the ground in such an exaggerated manner as actually to interfere 
with the view farther ahead. With the beam thrown out in a horizontal direction any obstruction in the road can be seen at a distance of 150 to 2,00 yards on a dull, but adequately illuminated, track.

Complicated devices of the nature of prismatic front attachments, etc., which have been advertised by various British and American makers, do not overcome the evils of this restriction. In fact, if regulations of this kind should be persisted in, there would be no object in constructing any good and expensive brightly shining headlights, since any common lamp would be good enough for lighting up near objects. Under these conditions it becomes impossible to travel fast at night.

The following is a brief description of the headlights made at the Zeiss Works at Jena. These have a device by means of which the glare may be cut off by the driver and the light dimmed to the amount necessary for slow running. The driver should be under legal compulsion to cut off the full glare in lighted localities and when meeting vehicles carrying lights.

The design of the headlights should fulfil the following essential conditions:

They should be capable of throwing a beam of light a long way ahead on a country road. They should furnish an adequate illumination to either side for negotiating curves, and they should be provided with means for cutting off the glare at any time and instantaneously.

The intense light required for fast travelling on country roads is not attainable with the acetylene lamps fitted with lenses and sheet metal reflectors, as hitherto in use. It became necessary to employ lamps constructed on searchlight principles in such a way that the whole of the light furnished by the available source of light may be emitted by the optical component in the form of a beam of parallel rays. This secures the highest degree of intensity. It is not possible to attain this end with the aid of a combination of lenses, to say nothing of the large proportion of light which is absorbed by thick lenses. The desired result is best accomplished by the use of a parabolic reflector, thanks to the well-known property whereby it gathers all rays proceeding from a luminous point situated in its focal plane and sends them forth in the form of a beam of parallel rays of a diameter equal to that of the reflector. Now, all sources of light embrace a more or less extensive areathey are not points-so that every point constitutes a focus whence a parallel beam proceeds by reflection at the angle with respect to the optic axis at which the ray proceeding from the luminous point meets the apex of the reflector. Hence the larger the source of light and the shorter the focal length of the mirror, the greater will be the angle of the emitted outer beams of light with respect to the axis, that is, the greater will be the dispersion. Now, in order that the dispersion may be sufficient for taking curves, reflectors have been made with their focal lengths as short as possible. Also, to collect the utmost amount of light, reflectors have been given an extremely deep section. As a result the light is dispersed, not only to either side, where it is needed, but also in upward and downward directions, where it fulfils no purpose. Moreover, the light which emerges in front, without being reflected and collected by the mirror, is scattered in all directions. 
Messrs Zeiss were the first to design projector lights in which these defects have been overcome, in the first place by giving the reflectors a much flatter profile and a longer focus. In these lamps the light, which in the older headlights shone directly forward, is rendered available for reflection by the parabolic mirror by placing a small spherical mirror in front. One of these earlier headlights is shown in Fig. $\mathrm{I}$.

Naturally, these reflectors furnish a far more intense light, since the whole of the light is collected and concentrated within a relatively small space. The beam which they form is therefore best adapted for lighting when travelling along a straight track at a maximum speed.

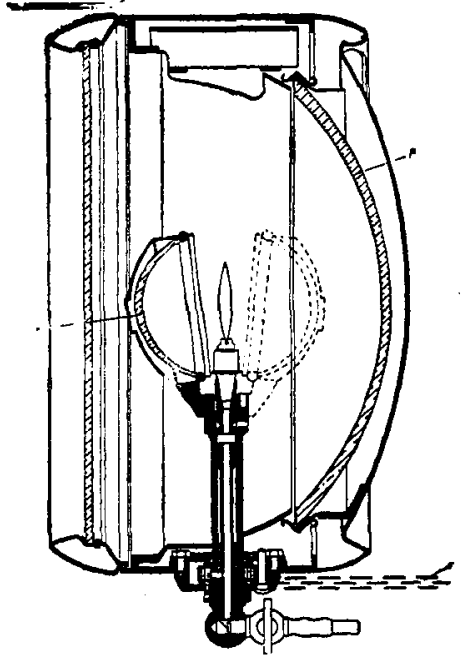

Fig. I

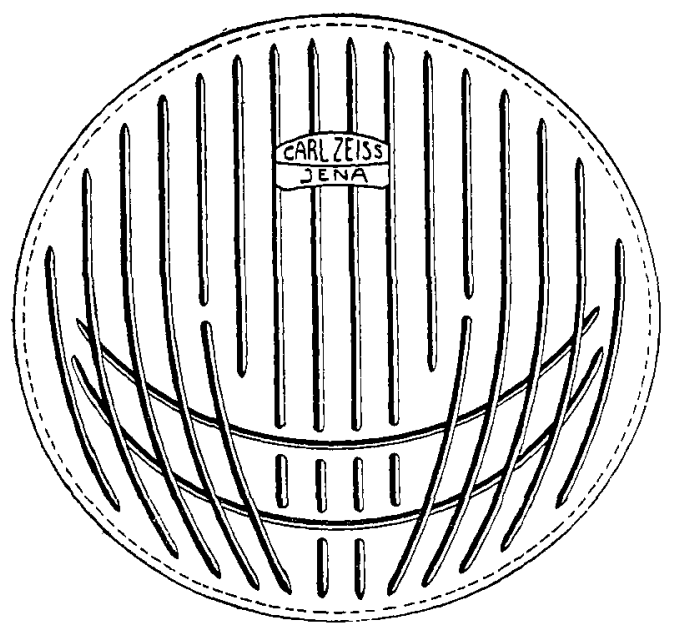

Fig. 2

A different arrangement was needed for the negotiation of curves. In this case a beam lighting up a long track would be most undesirable; moreover, the brightness in the curves may be much less than at the middle. The lamps were accordingly fitted with glass fronts provided with specially formed prismatic ribbing (Figs. 2, 3) by the action of which a small portion of the full light is deflected to the right or left as well as in a downward direction. The extent of this deflection is just sufficient to cover the curve and to provide the requisite amount of lighting in front. By this means the whole of the available light is accordingly collected and directed in appropriate quantity where it is required for taking the curve. No light is lost, and the intensity of the main body of the light is lessened by an inappreciable amount. The shallow form of the reflector has rendered it possible to make it of glass instead of sheet metal, as in the older headlights. The glass reflector has the advantage that it may be endowed with a much higher polish than is possible in the case of sheet metal mirrors. This in itself conduces to a greater intensity of the light. 
The life of the silvering is almost unlimited, since the glass is silvered at the back. The coat of silver is further protected by a deposit of copper and an application of varnish.

The reason why glass reflectors are not used universally is to be found in the fact that they are far more difficult to make than other kinds of reflectors. Metal reflectors may be drawn, spun, or cast, whereas glass reflectors require to be sunk and then ground and polished according to optical standards. It is an easy matter to grind a spherical reflector, but it is a laborious business to achieve a good parabolic surface and, moreover, the difficulty increases with the depth of the mirror.

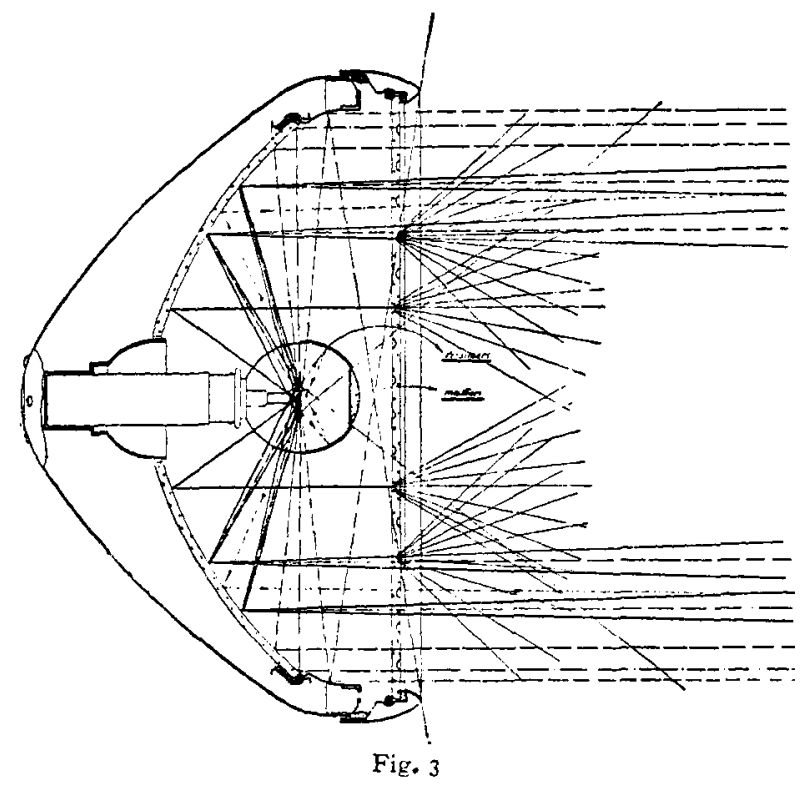

Truly faultless mirrors of great depth are necessarily too costly for use in headlights. At the Zeiss works the angle of aperture of the reflectors is not allowed to exceed $180^{\circ}$, but within this limit the mirrors are sunk, ground, and polished to satisfy exacting optical requirements.

The extensive use of sheet metal reflectors, and even mirrors made of ceramic materials coated with silver foil, is only justified by the fact that they are much cheaper to produce. It cannot be denied that very excellent mirrors are made of sheet metal. Unfortunately, sheet metal reflectors have the disadvantage of being extremely sensitive, so much so that they may barely be touched without an immediate risk of nasty patches forming on their surface; these cannot be removed, for the mirrors may not be cleaned with a polishing cloth, since the silvering, just like the silver foil, requires to be protected from oxidation by a coating of colourless lacquer. Despite this protective coating the reflecting capacity of these mirrors declines very soon. 
Mirrors silvered in front have undoubtedly a very high reflecting capacity, but this lasts for a short time only. In many cases the reflectors are permanently attached to the glass front for the better protection of the silvering. The chauffeur has then no access to the reflecting surface. In the event of the glass front being shattered, which happens often enough, the mirror would more likely than not be destroyed as well.

Fig. I shows a Zeiss acetylene headlight. The flame is at the principal focus of the large parabolic reflector and at the centre of the spherical mirror. Fig. 4 shows the path of the rays within the reflector. The dimming effect is obtained by turning the small spherical mirror through an angle of $\mathrm{I} 80^{\circ}$ about the axis of the flame. This cuts the light off from the parabolic mirror, and the flame together with the small

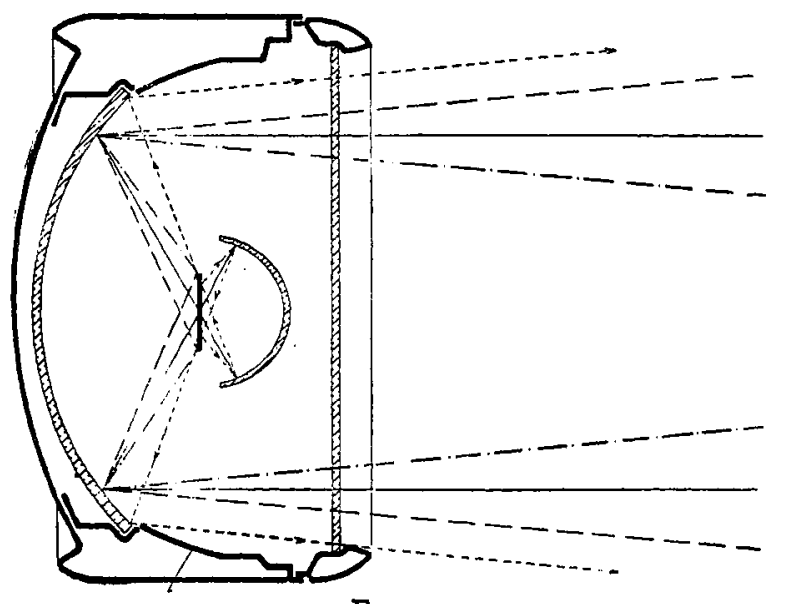

Fig. 4

spherical mirror will now light up the track in front of the car (Fig. 5). Headlights of this type have been made by Messrs Zeiss for the last twelve years.

Fig. 6 shows a Zeiss electric headlight. It will be seen that the mirror encloses one half of the incandescent lamp bulb. The light which in the ordinary course would pass out in front is intercepted by the silvered front half of the bulb. The latter throws back the whole of this light, as in the case of the acetylene lamp, upon the parabolic reflector, so that the whole of the light furnished by the incandescent lamp is turned to account. The central portion of the bulb corresponding to the size of the lamp fitting is left unsilvered and is frosted, so that the light which otherwise would be idly reflected back upon the fitting may emerge in front and is thus rendered available for lighting up the track.

An important feature of the Zeiss headlights consists in the focussing arrangement to the incandescent lamp. Unfortunately these lamps, as supplied by their makers, are not always sufficiently uniform to ensure a complete use of light under all circumstances. It was therefore found desirable to provide the lamp with a focussing adjustment. 


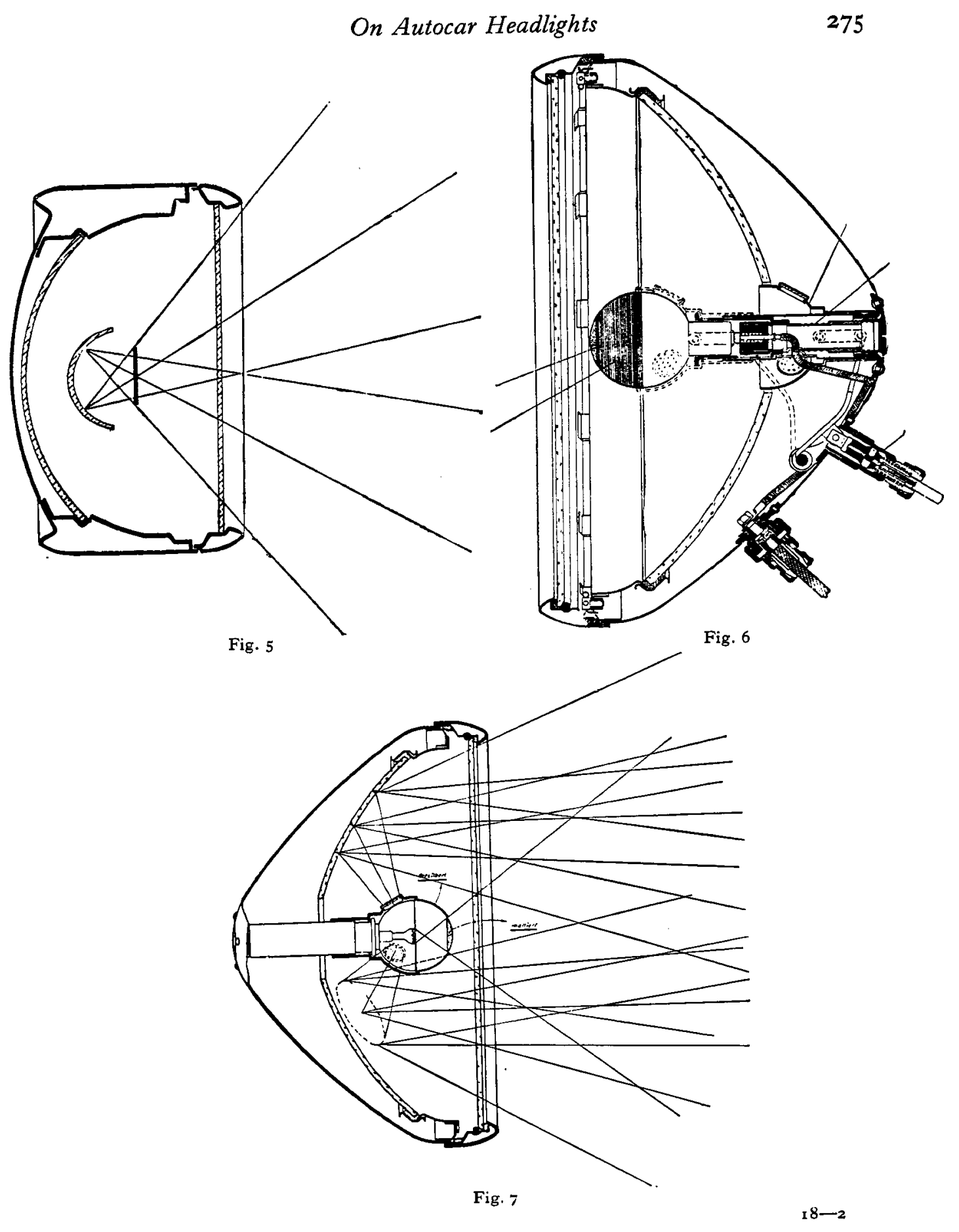


In the new Zeiss headlights dimming is effected, through the agency of a Bowden wire, by pushing a hemispherical cup of sheet metal from the back over the bulb, whereby the light is cut off from the reflector. In order that there may be sufficient light for driving through towns, the cup is fitted with three small densely frosted glass windows (Fig. 7). If sufficiently opalised, these windows behave like self-luminous sources of light. There is therefore no glaring effect, since the windows are not in the focal plane of the mirror. In fact, the effect produced within the headlight is that of three greatly magnified images of the windows, which adequately light up the track without producing any glare whatever. Nevertheless, the light thrown on the track is much brighter than that obtainable by the use of a small lamp at the side or in the upper part of the headlight, in that the presence of the three small windows ensures three times the amount of light.

The consumption of current is naturally the same in dimmed and in undimmed headlights, which is frequently regarded as an objectionable anomaly. The answer to this objection is that it is quite immaterial, so long as the dynamo is running, as to whether a little more or less current is absorbed. It is only with the dynamo out of action, when stopping in the road, that the larger amount of current used becomes a matter to be considered, since it involves a heavier strain upon the battery. But even then there is, generally speaking, no actua! loss, since car batteries are notoriously far more often damaged by overcharging than by being allowed to run down. In the majority of cases motorists do not completely discharge their batteries, and yet it is absolutely essential for the welfare of the battery that this should be done periodically. Those, however, who insist on economising battery current are at liberty to light their cars during waiting periods, with the motor standing, by means of two very small lamps of the type of number plate lamps at the sides of the mud guards, switched on in place of the headlights. As stationary lights these lamps fully satisfy all existing regulations.

\title{
TWO IMPROVED MOTOR HEADLAMPS
}

\author{
By E. CULVER. \\ (The United Kingdom Optical Co., Ltd.)
}

THE first lamp has been designed to enable a driver to see well ahead and yet not to inconvenience others. Almost all of the light is collected and projected straight ahead, or, by the use of modified fronts, part or all can be spread out sideways if desired. In the lamp exhibited part of the light is spread sideways to illuminate the width of the road without causing dazzle, whilst a very bright light is projected forward with a small dispersion sideways.

This is effected by the combination, in a novel manner, of certain well-known optical parts, viz.:

(I) a spherical reflector,

(2) an improved condenser or bull's eye lens, 